\title{
La loi sur la vasectomie est peu connue
}

\author{
Mohammed BADRAOUI, Franck BRUYERE, Yves LANSON
}

Service d'Urologie, CHU Bretonneau, Tours

\section{RESUME}

Objectif : connaître les pratiques sur la vasectomie et les connaissances de la loi qui les régit.

Matériel : un questionnaire de 17 items fut distribué à 262 chirurgiens susceptibles de réaliser des vasectomies dont 149 urologues et 68 gynécologues.

Résultats : Soixante et un pour cent des interrogés ont eu des demandes de vasectomies, $40 \%$ les pratiquent dont $60 \%$ en ambulatoire, $29 \%$ en hospitalisation et $11 \%$ en consultation.

Cinquante cinq pour cent pensent que la vasectomie est couverte par les assurances médicales, et $60 \%$ pensent abusivement qu'une déclaration au conseil de l'ordre des médecins est obligatoire.

Vingt-neuf pour cent ignorent l'obligation de l'information écrite au patient.

Vingt-huit pour cent connaissent le délai de réflexion de 4 mois avant le geste seulement et $\mathbf{4 5 \%}$ l'obligation du consentement écrit.

Conclusion : dans cette enquête, $14 \%$ des praticiens connaissent à la fois le délai de réflexion de 4 mois, le consentement et l'information écrits obligatoires. La loi régissant la stérilisation mérite d'être beaucoup plus largement diffusée.
Mots clés : contraception masculine, vasectomie, évaluation des pratiques urologiques

\section{INTRODUCTION}

Urologues comme gynécologues sont les praticiens les plus à même de réaliser des vasectomies. Avant la loi de juillet 2001 fixant les normes légales de la stérilisation masculine ou féminine, la majorité des ces spécialistes refusaient la pratique de ce geste par peur de non couverture par les sociétés d'assurances professionnelles. Une année et demie après la parution de la loi (voir Annexe 1), nous voulions savoir le degré de sa connaissance par les urologues, qu'ils fassent ou non des vasectomies, et faire une évaluation comparative dans une population de gynécologues puisque la loi est la même pour la vasectomie et la stérilisation tubaire.

\section{MATERIEL ET METHODES}

C'est une enquête qui a eu lieu entre janvier et juin 2003.

Un questionnaire (voir Annexe 2) de 17 items fut distribué à 262 chirurgiens dont 149 urologues et 68 gynécologues. Cette distribution fut réalisée au cours de congrès ou réunions de spécialités, soit à Paris, soit dans la région Ouest de la France. Les participants étaient avertis au cours de la réunion qu'une enquête sur la vasectomie allait leur être proposée. Seuls les spécialistes d'accord pour participer à l'enquête ont eu un questionnaire et l'on rempli.

Nos résultats ont été analysés en utilisant le test de chi2 chaque fois que l'effectif de l'échantillon nous a permis de faire une comparaison statistiquement significative.

Correspondance

Dr Mohammed BADRAOUI - service d'urologie, $\mathrm{CHU}$ Bretonneau, 2 bd Tonnellé, 37044 Tours -

Tel 06.15.26.75.97 - Fax 02.47.47.69.91 -

Email : badraouimed@yahoo.fr 


\section{RESULTATS}

Deux cent soixante deux praticiens ont répondu à notre questionnaire, allant des jeunes diplômés aux spécialistes de longue date. La majorité d'entre eux appartenait à une tranche d'âge allant de 36 à 55 ans (Tableau 1).

Tableau 1 : répartition en fonction de l'âge des praticiens interrogés.

\begin{tabular}{lcc}
\hline Age (ans) & nombre & $\%$ \\
\hline$<30$ & 31 & 11,8 \\
$30-35$ & 41 & 15,7 \\
$36-45$ & 72 & 27,5 \\
$46-55$ & 69 & 26,3 \\
$>55$ & 49 & 18,7 \\
\hline
\end{tabular}

Les professeurs universitaires (PUPH) représentent 12,2\% des praticiens intérrogés et $21 \%$ des urologues. Les 32 professeurs ayant répondu représentent $37 \%$ des enseignants d'urologie des facultés françaises.

Soixante pour cent des praticiens ayant répondu à notre questionnaire ont déjà eu une demande de vasectomie et $40 \%$ la pratiquent $(60 \%$ en ambulatoire, $29 \%$ en hospitalisation et $11 \%$ en consultation).

Plus de la moitié des intérrogés pensent que la vasectomie est couverte par la sécurité sociale et $18 \%$ pensent qu'une décalaration au conseil de l'ordre des médecins est obligatoire.

Vingt neuf pour cent des interrogés ignorent l'obligation de l'information écrite au patient. Seuls $27 \%$ des interrogés connaissent le délai de réflexion de 4 mois avant le geste, et $45 \%$ l'obligation du consentement écrit.

Seuls $14 \%$ des interrogés connaissent ces trois items pourtant très précis de la loi (obligation de l'information écrite, délai de réflexion de 4 mois, obligation du consentement écrit). Parmi ces rares praticiens qui connaissent les trois items importants de la loi, $53 \%$ sont des urologues (dont $11 \%$ sont des PUPH), et un sur deux parmi eux a fait des vasectomies. Statistiquement, les praticiens qui font la vasectomie ne connaissent pas mieux la loi que ceux qui ne la pratiquent pas.

Parmi les gynécologues, $30 \%$ connaissent les trois items importants de la loi pour $17 \%$ des urologues.

Quarante huit praticiens, soit $18,3 \%$, ne connaissent aucun item de la loi ; parmi eux 33 urologues (parmi lesquels 9 PUPH), dont 18 ont déjà fait des vasectomies.

Pour la connaissance de la loi, on n'a pas noté de différence significative par rapport à l'âge des praticiens.

\section{DISCUSSION}

\section{Représentativité des échantillons évalués au sein des spécialistes}

Les urologues ayant répondu à l'enquête représentent $15 \%$ des urologues français.

Par ailleurs, les urologues libéraux sont en France les plus nombreux alors qu'ils ne sont que $25 \%$ dans l'étude. En revanche, les professeurs d'urologie constituent $8 \%$ des urologues français et représentent ici $21 \%$ des réponses.

Les gynécologues ayant participé à l'enquête représentent un pourcentage beaucoup plus faible de l'ensemble de ces spécialistes. Pour eux, la représentativité n'était pas un objectif. II nous semblait souhaitable d'avoir seulement un petit échantillon à comparer aux données urologiques.

II est difficile de savoir si des échantillons plus représentatifs de l'urologie française auraient modifié les données.

\section{Pratique des vasectomies}

II est impossible sur ces seules données d'extrapoler à l'ensemble des urologues de manière chiffrée. On peut cependant constater qu'une majorité d'urologues ont des demandes de vasectomie mais que parmi ceux-ci, il persiste environ 4 praticiens sur 10 qui ne la réalisent pas, 18 mois après la parution des textes de loi.

\section{Connaissance de la loi}

Les trois items les plus importants de la loi (l'information écrite au patient, le délai de réflexion de 4 mois et le consentement écrit du patient) sont très rarement connus par les praticiens. II n'est pas possible d'extrapoler à l'ensemble des urologues et des gynécologues le fait que $14 \%$ seulement connaissent les articles les plus importants de la loi. On peut cependant dire que si parmi l'échantillon de $15 \%$ des urologues et de $37 \%$ des professeurs d'urologie cette loi est si mal connue, il est probable que l'ensemble de tous les spécialistes la connaisse très mal également.

La contraception et la stérilisation sont au programme du concours national classant qui permettra à tous les étudiants de choisir leur spécialité et leur lieu de formation à partir de novembre 2004. Si aucun changement ne se fait, on peut se demander si les étudiants connaîtront mieux cette loi que les enseignants qui sont censés leur apprendre.

Les gynécologues, plus souvent confrontés aux demandes de stérilisation, connaissent mieux les trois items de la loi que les urologues (30\% versus $17 \%$ respectivement). II est possible qu'ils aient eu, par les contraintes de la stérilisation tubaire, une meilleure écoute lors de la parution de la loi, ou bien les sociétés savantes ont assuré une meilleure diffusion de l'information.

Les praticiens qui font la vasectomie ne connaissent pas mieux la loi par rapport à ceux qui ne la pratiquent pas.

Cette ignorance de la loi peut avoir des conséquences juri- 
diques graves mettant en erreur les praticiens puisque la non connaissance des impératifs légaux entraîne obligatoirement une mauvaise information des demandeurs, puis des pratiques non conformes.

\section{CONCLUSION}

Notre travail a porté sur un petit échantillon d'où la difficulté d'extrapoler à la population générale des urologues. En revanche, on peut dire que d'une part, malgré la loi, un certain nombre de praticiens ont des demandes de stérilisation mais qu'une partie d'entre eux ne les réalisent pas, et d'autre part que la loi sur la vasectomie n'est pas bien connue y compris par les chirurgiens qui la pratiquent.

Nous voulons, à travers cette enquête, contribuer à la connaissance de cette loi et à sa diffusion.

\section{REFERENCES}

Code de la santé publique, art. L2123-1. Loi n ${ }^{\circ} 2001-588 \mathrm{du}$ 4 juillet 2001. Art.26 journal officiel du 7 juillet 2001.

Communication au $X X^{\circ}$ Congrès de la Société d'Andrologie de Langue Française, Orléans, 11-13 Décembre 2003.

Manuscrit reçu : juin 2004 ; accepté août 2004.

\author{
ABSTRACT \\ The vasectomy law is not well known

\begin{abstract}
Mohammed BADRAOUI, Franck BRUYERE, Yves LANSON
\end{abstract}

Objective: To determine vasectomy practices and knowledge of the law governing these procedures.

Material: A 17-item questionnaire was sent to 262 surgeons likely to perform vasectomies, including 149 urologists and 68 gynaecologists.

Results: Sixty one per cent of doctors had received requests for vasectomy and $\mathbf{4 0} \%$ performed this procedure, as an outpatient procedure in $60 \%$ of cases, in hospital in $29 \%$ of cases and as an office procedure in $11 \%$ of cases.

Fifty five per cent of doctors thought that vasectomy was covered by medical insurance, and $60 \%$ wrongly believed that a declaration must be made to the Ordre des Médecins (Medical Board).

Twenty nine per cent were not aware of the need for written information of the patient.

Only $28 \%$ were aware of the 4 -month period of reflection before the procedure and $45 \%$ were aware of the need for written consent.

Conclusion: in this survey, $14 \%$ of practitioners were simultaneously aware of the need for a 4-month period of reflection, consent and written information. Wider diffusion of information about the law governing sterilization is required.

Key words : male contraception, vasectomy, assessment of urological practices 


\section{Annexe 1 \\ Texte de loi sur l'interruption volontaire de grossesse}

Dans le cadre de la loi $n^{\circ} 2001-588$ du 4 juillet 2001 relative à l'interruption volontaire de grossesse et de la contraception parue au J.O. Numéro 156 du 7 juillet 2001 page 10823, l'Assemblée nationale a autorisé la stérilisation à visée contraceptive, qui était jusqu'alors interdite en France, bien que le Conseil national de l'ordre des médecins ait plaidé en sa faveur dès 1996.

Loi $n^{\circ} 2001-588$ du 4 juillet 2001 relative à l'interruption volontaire de grossesse et à la contraception.

\section{Article 26:}

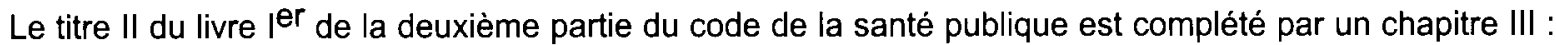

"stérilisation à visée contraceptive" :

Art. L.2123-1 : La ligature des trompes ou des canaux déférents à visée contraceptive ne peut être pratiquée sur une personne mineure. Elle ne peut être pratiquée que si la personne majeure intéressée a exprimé une volonté libre, motivée et délibérée en considération d'une information claire et complète sur ses conséquences.

" cet acte chirurgical ne peut être pratiqué que dans un établissement de santé et après une consultation auprés d'un médecin.

" ce médecin doit au cours de la première consultation :

- informer la personne des risques médicaux qu'elle encourt et des conséquences de l'intervention.

- lui remettre un dossier d'information écrit.

“il ne peut être procédé à l'intervention qu'à l'issue d'un délai de réflexion de quatre mois après la première consultation médicale et après une confirmation écrite par la personne concernée de sa volonté de subir une intervention.

“ un médecin n'est jamais tenu de pratiquer cet acte à visée contraceptive mais il doit informer l'intéressé de son refus dès la première consultation. "

\section{Article 27 :}

Après l'article L.2123-1 du même code, il est inséré un article L. 2123-2 ainsi rédigé :

Art. L. 2123-2 : la ligature des trompes ou des canaux déférents à visée contraceptive ne peut être pratiquée sur une personne mineure. Elle ne peut être pratiquée sur une personne majeure dont l'altération des facultés mentales constitue un handicap et a justifié son placement sous tutelle ou sous curatelle que lorsqu'il existe une contre-indication médicale absolue aux méthodes de contraception ou une impossibilité avérée de les mettre en œuvre efficacement.

"I'intervention est subordonnée à une décision de juge des tutelles saisi par une personne concernée, les père et mère ou le représentant légal de la personne concernée.

"le juge se prononce après avoir entendu la personne concernée. Si elle est apte à exprimer sa volonté, son consentement doit être systématiquement recherché et pris en compte après que lui a été donné une information adaptée à son degré de compréhension. II ne peut être passé outre à son refus ou à la révocation de son consentement.

"le juge entend les père et mère de la personne concernée ou son représentant légal ainsi que toute personne dont l'audition lui paraît utile.

" il recueille l'avis d'un comité d'experts composé de personnes qualifiées sur le plan médical et de représentants d'associations de personnes handicapées. Ce comité apprécie la justification médicale de l'intervention, ses risques ainsi que ses conséquences normalement prévisibles sur le plan physique et psychologique.

" un décret en Conseil d'Etat fixe les conditions d'apolication du présent article." 
Annexe 2

ENQUETE VASECTOMIE

Vous êtes : Exercice principal

Hôpital

Privé

Gynécologue

Urologue

Age : $\quad 30-35$ ans

36-45 ans

46-55 ans

$>55$ ans

Vous avez parfois des demandes de vasectomie :

Oui

Non $\square$

Si oui : $\quad$ En consultation

En ambulatoire

En hospitalisation

Si vous les refusez c'est :

Parce qu'interdites

Problème éthique

Autre

D'après vos connaissance, la vasectomie est couverte par les assurances

médicales :

(si vous ne savez pas, ne cocher aucune case)

Oui $\square$

Non $\square$

La vasectomie est possible par tous les chirurgiens :

Oui

Non $\square$

(pas seulement pour certaines spécialités)

La vasectomie impose une déclaration à l'ordre des médecins : $\quad$ Oui $\square \quad$ Non $\square$

La vasectomie peut être réalisée après déclaration à l'assurance responsabilité

civile professionnelle :

Oui

Non $\square$

La vasectomie impose une information médicale écrite :

Oui $\square$

Non $\square$

La réalisation de la vasectomie impose un délai de réflexion après information :

Non

1 mois

2 mois

3 mois

4 mois

Un consentement écrit du malade est :

Non obligatoire $\square$

Souhaitable

Obligatoire

Un chirurgien peut refuser une vasectomie :

Oui

Non $\square$

S’il refuse il est tenu à donner des références d'autres chỉrurgiens :

Oui $\square$

Non $\square$ 